\section{Rational prescribing: the principles of \\ drug selection}

Simon Maxwell, Consultant Physician and Clinical Pharmacologist, Clinical Pharmacology Unit, University of Edinburgh

Prescribing is a complex task requiring:

- diagnostic skills

- knowledge of medicines

- an understanding of the principles of clinical pharmacology

- communication skills

- appreciation of risk and uncertainty.

The accumulation of clinical trials' data on modern therapies might have been expected to provide sufficient evidence to support most clinical decisions. In fact, clinicians prescribe in varied circumstances, often in the absence of evidence, and rational prescribing decisions must be based on knowledge interpreted in the light of many other factors.

\section{Rational prescribing}

Rational prescribers should attempt to:

- maximise clinical effectiveness

- minimise harms

- avoid wasting scarce healthcare resources

- respect patient choice.

Rational prescribing normally follows a logical sequence from diagnosis to follow-up (Fig 1).

\section{Diagnosis}

Prescribing decisions should be based on the primary diagnosis and relevant secondary diagnoses. Ideally, these should have been made or confirmed by the prescriber who will take responsibility for the effects of treatment. Appreciating that diagnoses are made with varying degrees of uncertainty is important when assessing the benefit-to-harm balance of treatment. For instance, antibiotics are often prescribed on the basis of presumed antibacterial sensitivity with the expectation of significant benefit. However, this can expose the recipient to harm without the prospect of cure.

\section{Prognosis}

The prognoses of the primary and secondary diagnoses will affect rational treatment choices. A secondary diagnosis with a poor prognosis, such as lung cancer, will severely limit the benefits of treating a primary one, such as hypercholesterolaemia. On the other hand, the excellent prognosis of influenza in a healthy adult limits the potential benefits of antiviral therapy.

\section{Goals of therapy}

Goals of therapy may include:

- curing a disease (eg cancer, infection)

- relieving symptoms without affecting the underlying condition (eg headache, diarrhoea)

- combining two outcomes (eg inflammatory bowel disease and arthritis)

- long-term prevention (eg hypertension, osteoporosis)
- replacing deficiencies (eg hypothyroidism), and occasionally

- therapeutic trials to aid diagnosis.

\section{Treatment selection}

Prescribers are commonly faced with more than one choice of treatment, including non-pharmacological therapies or no treatment. For example, the management of arthritis might include reassurance, simple analgesia, physiotherapy, non-steroidal anti-inflammatory drugs, disease-modifying antirheumatic drugs, intra-articular steroids or surgery.

\section{Monitoring}

Each prescription constitutes an experiment the outcome of which is never certain. It is therefore important to monitor the effects of treatment, re-evaluate the benefit-harm balance and, if indicated, withdraw the drug or change the dose. The most appropriate end-point will be objective assessment of the clinical outcome (eg recovery from pneumonia), but assessment may be subjective (eg pain relief, improved quality of life). Patient satisfaction is also important. Sometimes

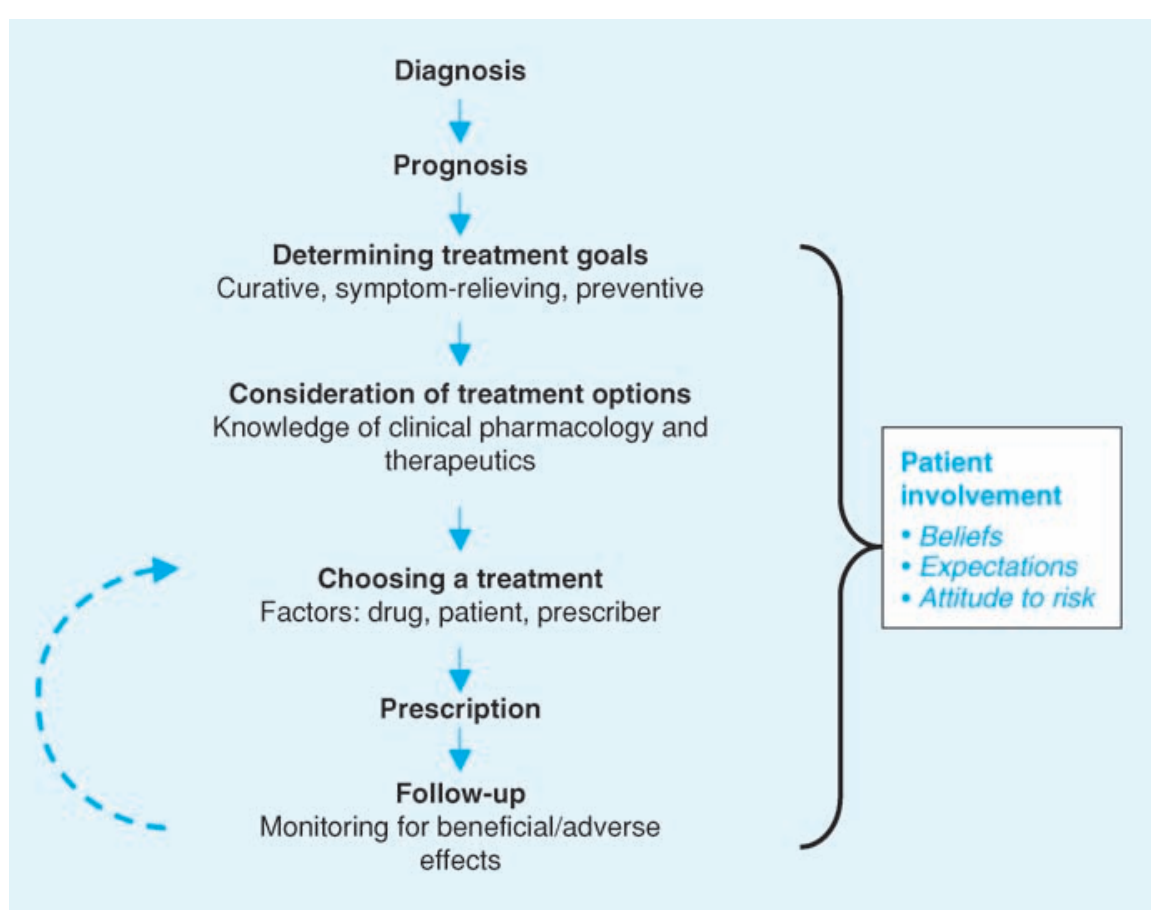

Fig 1. The process of rational prescribing. 
the outcome is difficult to measure (eg management of epilepsy) or requires long-term follow-up (eg preservation of health in HIV infection). In such cases, validated surrogate markers (eg serum anticonvulsant concentration, CD4 cell count) may guide therapy. Adverse events can also be monitored in different ways.

\section{Partnership with patients}

Patients make important contributions to rational prescribing decisions. Their beliefs and expectations affect the goals of therapy and help in judging the acceptable benefit-harm balance when selecting treatments. They will often play a key role in monitoring treatment, not least by providing early warning of adverse events. Patients involved in clear communication with prescribers concerning reasons for drug selection, goals, duration of treatment and potential adverse effects have improved compliance, more confidence in prescribers and greater satisfaction with healthcare services. Thus, whenever possible, patients should be fully informed about their medicines (Table 1).

\section{Drug and dose selection}

Having considered diagnosis, prognosis and goals of therapy, prescribers often select from several pharmacological options. The best choice should max-

Table 1. What patients need to know about their medicines.

- The reason for taking the medicine

- How the medicine works

- How to take the medicine

- What benefits to expect (how to know if it works)

- What adverse effects might occur:

- common

- serious

- Precautions that improve safety:

- symptoms to report

- monitoring required

- potential drug-drug interactions

- When to return for review imise the benefit-harm balance based on drug and patient factors, taking into account restrictions based on availability and costs (Table 2).

\section{Drug factors influencing drug selection}

\section{Pharmacokinetics}

Drugs in the same class (or different formulations of the same drug) may have different bioavailability, doseconcentration curves and half-lives. These factors will determine the dosing schedule. Once-daily dosing is convenient and encourages adherence. Pharmacokinetic characteristics may also influence interindividual variability in dosage requirements. For example, some drugs:

- differ with respect to their specificity for the target organ
- reach tissues (eg the brain) to cause adverse effects

- are metabolised in the liver or excreted - important in patients with hepatic or renal impairment

- are more likely to cause drug interactions by cytochrome P450 inhibition (eg simvastatin versus pravastatin).

\section{Pharmacodynamics}

A drug with a low therapeutic index (the ratio between the dose required to cause adverse effects and that required for efficacy) is less favourable if alternatives exist. Similarly, the steepness of the doseresponse curve will influence the ease with which the dose can be optimally titrated. Selectivity for a receptor subtype may be relevant when choosing drugs

Table 2. Factors that influence rational drug and dosage selection.

\begin{tabular}{|c|c|}
\hline Diagnosis & $\begin{array}{l}\text { Primary: condition to be treated } \\
\text { Secondary: other conditions that may influence the benefit- } \\
\text { to-harm balance }\end{array}$ \\
\hline Prognosis & Influences the likely duration of benefits and harms of treatment \\
\hline \multicolumn{2}{|l|}{ Drug factors: } \\
\hline Pharmacokinetic & $\begin{array}{l}\text { Frequency of dosing: influences adherence } \\
\text { Bioavailability: if consistent, makes drug response more predictable } \\
\text { Tissue distribution: can affect adverse effects at sites other than } \\
\text { targeted } \\
\text { Routes of metabolism/excretion: increased anticipation of } \\
\text { variable response } \\
\text { Drug interactions: greater safety if less frequent }\end{array}$ \\
\hline Pharmacodynamic & $\begin{array}{l}\text { Target specificity and selectivity: influences likelihood of adverse } \\
\text { effects } \\
\text { Dose-response characteristics: influences ease of dose titration } \\
\text { Therapeutic index: influences ease of dose selection }\end{array}$ \\
\hline Therapeutic & $\begin{array}{l}\text { Efficacy in relieving symptoms } \\
\text { Efficacy on morbidity/mortality/hospitalisation } \\
\text { Impact on disease progression (eg prolongation of life) }\end{array}$ \\
\hline Safety & $\begin{array}{l}\text { Frequency of adverse effects } \\
\text { Seriousness of adverse effects (eg allergy, idiosyncratic reactions) } \\
\text { Ease with which adverse effects can be predicted, monitored } \\
\text { and prevented }\end{array}$ \\
\hline Cost & Availability of alternatives with similar efficacy \\
\hline Patient factors & $\begin{array}{l}\text { Health beliefs and attitude to risk } \\
\text { History of previous adverse drug reactions } \\
\text { Vulnerability to adverse effects (eg organ damage, reduced } \\
\text { physiological reserve) } \\
\text { Current drug therapy including interacting drugs } \\
\text { Likely adherence to therapy or follow-up monitoring }\end{array}$ \\
\hline Prescriber factors & $\begin{array}{l}\text { Familiarity with prescribing choices } \\
\text { Ease of follow-up: may depend on resources }\end{array}$ \\
\hline
\end{tabular}


that avoid predictable adverse effects. Some drugs require more complex monitoring, which can affect costs and patient time (eg warfarin versus aspirin).

\section{Therapeutic impact and safety}

A drug may be more efficacious in relieving symptoms, improving surrogate markers or preventing clinical events (eg morbidity, mortality, hospitalisation) or have fewer and less serious adverse effects (eg carbamazepine $v$ phenytoin). Large randomised controlled trials (RCTs) are considered the optimal sources of evidence, but extrapolating the results to prescribing decisions in the real world requires caution. RCTs usually recruit highly selected participants (eg based on age or disease severity) without comorbidities or receiving interacting drugs. Such additional factors can influence efficacy or adverse outcomes, potentially reducing the former and enhancing the latter, thus limiting the external validity of RCTs.

\section{Costs}

All healthcare systems have limited resources. The rapidly increasing cost of medicines forces all prescribers to consider cost-effectiveness as a factor in drug selection. This is taken into account when devising local formularies and in the decisions of the National Institute for Health and Clinical Excellence. Perhaps the most obvious example of cost-effective prescribing is selecting a generic rather than a branded drug from the same class. However, cost may be outweighed by other factors, notably significant differences in efficacy or safety. (See accompanying article on pharmacoeconomics.)

\section{Patient factors influencing drug selection}

\section{Previous adverse drug reactions}

Knowledge of previous adverse reactions will affect drug or dose selection but depends on taking a careful drug history. This is particularly important in the case of allergic reactions (eg beta-lactam antibiotics).

\section{Vulnerability to adverse effects}

Some patients will have organ damage that may affect drug choices. For instance, a beta-blocker for angina may be undesirable in patients with peripheral vascular disease or asthma but attractive in those with heart failure. Reduced physiological reserve increases the vulnerability of elderly patients to the adverse effects of many drugs (eg anticholinergics, central nervous system depressants, vasoactive drugs) and necessitates dosage reductions.

\section{Current drug therapy}

Any current drug therapy may affect drug or dosage selection, mainly because of potential drug interactions. For example, the dose of simvastatin should not be increased beyond $20 \mathrm{mg}$ nocte in patients taking amiodarone or verapamil because of the increased risk of muscle toxicity.

\section{Other patient factors}

The likelihood that patients will adhere to therapy or follow-up monitoring is important for drugs such as warfarin and insulin which have a low therapeutic index and where alternatives are less effective. Health beliefs and attitude to risk can influence the initial decision to prescribe or the choice of medicine. This is particularly obvious in long-term preventive therapy when benefits may be imperceptible. About half of patients adhere poorly to such treatments, emphasising the role of patient partnership in making rational prescribing decisions.

\section{Prescriber factors influencing drug selection}

\section{Familiarity}

Lack of familiarity of prescribers with medicines increases the chance of adverse outcomes, mandating continuing professional development. However, lack of experience should not impede the introduction of new, more rational prescribing practices.

\section{Ease of follow-up}

Some medicines require careful review and monitoring to ensure that safety is maximised or dose titration optimal. The ease with which these can be accomplished is important.

\section{Examples of irrational prescribing}

Rational prescribing aims to ensure that selection is not a simple formulaic linkage of drugs and doses to particular diagnoses, but involves individualising prescriptions as far as possible, taking account of the variables discussed above.

Table 3 offers some simple examples of irrational prescribing. They are illustrative only and do not acknowledge the complexity of real prescribing decisions. Prescribers commonly make probabilistic judgements that involve interpreting trial evidence in the light of specific circumstances such as patients' wishes, availability of resources and previous adverse events. For instance, more

\section{Key Points}

Prescribing is a complex task that requires interpretation of evidence from clinical trials in light of individual patient factors

Rational prescribing describes a logical approach that includes making a diagnosis, estimating prognosis, establishing the goals of therapy, selecting the most appropriate treatment and monitoring the effects of the treatment

Patients should be involved in several of these stages and their beliefs, expectations and attitudes to risk will contribute to rational prescribing decisions

Pharmacogenetics will help to individualise prescribing choices but will not replace the need for an understanding of the clinical pharmacology underpinning the selection of commonly prescribed drugs

KEY WORDS: drug selection, interindividual variation, monitoring therapy, rational prescribing 
expensive but equivalent medications may be justified if others have caused adverse effects or loss of confidence. Higher risk medicines may be acceptable if the potential benefit is estimated to be greater for an individual patient.

\section{Personalised medicines: the future?}

This article has discussed the traditional approach to prescribing in which individualised drug selection is based on evidence gathered from groups of similar patients mixed with best-guess judgements about the variability introduced by specific patient and drug factors. Recently, a new era of 'personalised' treatment has been predicted in which therapeutic choices will be individualised based on genetic variables affecting drug handling and action, allowing more specific prediction of outcomes. Indeed, pharmacogenetics is already being used to distinguish responders from nonresponders (eg trastuzumab for HER2-overexpressing breast cancer) and to avoid adverse effects (eg HLA $B \star 5701$ for abacavir hypersensitivity).
(See accompanying article on pharmacogenetics.)

The impact of this approach may however be limited because many of the variables outlined in Table 2 are not affected by genetics. This suggests that rational prescribing will remain based on a firm grounding in the principles of clinical pharmacology.

\section{Further reading}

1 Aronson JK. Balanced prescribing. Br J Clin Pharmacol 2006;62:629-32.

2 Aronson JK. Changing beta-blockers in heart failure: when is a class not a class? $\mathrm{Br}$ J Gen Pract 2008;58:387-9.

Table 3. Examples of irrational prescribing.

\section{Reason}

Low chance of benefit (compared with harm): Short-term conditions with good prognosis Preventive therapy in patients with poor prognosis conditions/poor quality of life Drugs used beyond the evidence base Dose too low

Wrong diagnosis

Increased risk of harm (compared with benefit): Vulnerability to adverse effects

$$
\begin{aligned}
& \text { Drug clearance altered } \\
& \text { Drug interactions likely } \\
& \text { Dose too high }
\end{aligned}
$$

Reduced adherence likely:

Too many medicines (polypharmacy) in patients with multiple conditions

Poor communication

\section{Unnecessary cost:}

Expensive drugs with no evidence of superior outcomes when cheaper drugs exist

Expensive drugs that offer slightly better outcomes at enormously increased cost

Drugs for ADRs:

Drugs prescribed to counteract the adverse effects of other medicines that could be replaced with suitable alternatives

\section{Example}

Antiviral drugs for influenza in healthy adults

Statin therapy in patients with a malignancy

Statin therapy for very young or very old patients

ACEls for CHF

Anti-anginal drugs prescribed for patients with GOR

Antibiotics for viral illnesses

Prescribing psychoactive medicines for elderly patient; NSAIDs for patients with impaired renal function; thromboprophylaxis in patients at risk of serious bleeding due to factors such as thrombocytopenia, peptic ulcer disease, coagulopathies, intracranial disease

Wrong doses in patients with renal or hepatic disease

Enzyme-inhibiting drugs in patients taking warfarin

Thiazide diuretics as antihypertensives

Aspirin for the prevention of cardiovascular disease

Prescribing all evidence-based therapies in elderly patients with chronic airways disease, hypertension, CHF, osteoporosis, GOR or RA

Antihypertensive drugs in young patients unclear about or unimpressed with the extent of likely benefit

Prescribing branded rather than generic statins in primary prevention

Some new therapies for cancer

Laxatives for verapamil-induced constipation

Salbutamol for beta-blocker-induced bronchospasm

Diuretics for amlodipine-induced ankle oedema

ACEI = angiotensin-converting enzyme inhibitor; $A D R=$ adverse drug reaction; CHF = chronic heart failure; GOR = gastro-oesophageal reflux; NSAID = nonsteroidal anti-inflammatory drug; $\mathrm{RA}=$ rheumatoid arthritis. 
3 Audit Commission. A prescription for improvement. Toward more rational prescribing in general practice. London: Stationery Office, 1994.

4 Barber N. What constitutes good prescribing? BMJ 1995;310:923-5.

5 Beers MH. Explicit criteria for determining potentially inappropriate medication use by the elderly. An update. Review. Arch Intern Med 1997;157:1531-6.

6 Blue JW, Colburn WA. Efficacy measures: surrogates or clinical outcomes? J Clin Pharmacol 1996;36:767-70.

7 Bucher HC, Guyatt GH, Cook DJ, Holbrook A, McAlister FA. Users' guides to the medical literature: XIX. Applying clinical trial results. A. How to use an article measuring the effect of an intervention on surrogate end points. Evidence-Based Medicine Working Group. JAMA 1999;282:771-8.

8 de Vries TP. Presenting clinical pharmacology and therapeutics: a problem based approach for choosing and prescribing drugs. Br J Clin Pharmacol 1993;35:581-6.

9 de Vries TP, Henning RH, Hogerzeil HV, Fresle DA. World Health Organization. Guide to good prescribing - a practical manual, 1994. WHO/DAP/94.11 www.who.int/ medicinedocs/en/d/Jwhozip23e/

10 Hogerzeil HV. Promoting rational prescribing: an international perspective. Review. Br J Clin Pharmacol 1995;39:1-6.

11 GP Notebook - a UK medical reference on the world wide web. Rational prescribing. www.gpnotebook.co.uk/simplepage.cfm?ID= 1362427972

12 Lesko LJ. Personalized medicine: elusive dream or imminent reality? Clin Pharmacol Ther 2007;81:807-16.

13 Osterberg L, Blaschke T. Adherence to medication. N Engl J Med 2005;353: 487-97.

14 Roland M, Torgerson D. Understanding controlled trials: what outcomes should be measured? BMJ 1998;317:1075.
15 Sackett DL, Rosenberg WM, Gray JA, Haynes RB, Richardson WS. Evidence based medicine: what it is and what it isn't. BMJ 1996;312:71-2.

16 Scott DK, Ferner RE. 'The strategy of desire' and rational prescribing. Br J Clin Pharmacol 1994;37:217-9.

17 Woodcock J. The prospects for 'personalized medicine' in drug development and drug therapy. Clin Pharmacol Ther 2007;81:164-9.

Address for correspondence:

Professor S Maxwell, Clinical

Pharmacology Unit, University of Edinburgh; Clinical Research Centre, Western General Hospital, Edinburgh EH4 2XU.

Email: s.maxwell@ed.ac.uk

\section{WORKING PARTY REPORTS}

\section{Acute medical care The right person, in the right setting - first time}

Acute medical services and the provision of acute medical care in our hospitals have evolved rapidly over the past decade. Acute medical emergencies are the most common reason for admission to an acute hospital, and acute medicine is the fastest growing medical specialty. Changes to the way acute medical services are delivered has been necessitated by a number of drivers, high among which are patient safety, improved quality of clinical care, clinical governance, and the need to train within the specialty.

Within our hospitals there is a need to reconfigure services to provide more efficient patient access to acute care - whenever that need arises. Acutely ill patients require rapid round-the-clock access to senior clinical decision makers, and to a nationally standardised approach to clinical assessment, documentation and illness management.

This report provides practical guidance for the delivery of acute medical services, identifying generic principles that can be configured to meet local needs. It recognises the important role that the multi-professional team plays in delivering a high-quality service. The report updates the 2004 report Acute medicine: making it work for patients and should be read by all those involved in delivering acute medical care and managing acute medical services.
Contents:

- Vision, remit and background

- Acute medical care

- Patient safety and clinical effectiveness

- Acute medical care within hospitals

- Workforce development, education and training

- References

- Recommendations

- Resources 